\title{
Cooperating Services in a Mobile Tourist Information System
}

\author{
Annika Hinze ${ }^{1}$ and George Buchanan ${ }^{2}$ \\ 1 University of Waikato, Hamilton, New Zealand, a.hinze@cs.waikato.ac.nz \\ ${ }^{2}$ UCL Interaction Centre, London, United Kingdom, g.buchanan@cs.ucl.ac.uk
}

\section{Introduction}

Complex information systems are increasingly required to support the flexible delivery of information to mobile devices. Studies of these devices in use have demonstrated that the information displayed to the user must be limited in size, focussed in content [1] and adaptable to the user's needs [2]. Furthermore, the presented information is often dynamic - even changing continuously. Eventbased communication provides strong support for selecting relevant information for dynamic information delivery.

We report about the extension of our mobile tourist information system TIP with cooperating services. We are building upon our first-generation stationary core system, TIP 1.0 [3]. Previous work focussed on the interplay of different event/information sources and the event-based information delivery.

The extended TIP architecture provides users with information from modular, cooperating, mobile services. Modular services can be used in addition to the core system, allowing the users to use different services for similar purposes interchangeably (e.g., for guidance information in maps or textual representation). Cooperating services exchange context data and information for the benefit of the system's users. Mobile services can be used on typical hand-held devices; preferably with little or no installation and maintenance overhead for the user.

\section{Issues Identified and Lessons Learned}

We have identified a number of difficulties and challenges for creating an eventbased communication framework for mobile systems.

Communication Protocols. The range of inter-process communication techniques that are available between processes running on the same mobile device are limited and rather restrictive. On the other hand, communication between a mobile device and the TIP server can have several forms. A global framework should to hide such implementation details from the different components of the system.

Service Composition. The final service provided to the user (e.g., a tourist guide with map, sight data and recommendations) is internally a composition of a variety of services. Services need to communicate both within the same 
machine and between computers, using thick- and thin-client scenarios, or hybrid approaches. A sound framework must support new types of services and alternative realizations of the same service.

Standards. We have created a framework in which mobile services cooperate, but further forms of co-operation and composition are needed, e.g., to support new inter-process communications. Implementation details, particularly issues of standardisation, continue to be relevant. In the map system, different mapping scales and notations are used by different map and information providers, and further services must be introduced to mediate between systems that function in different notational standards.

As information systems move onto mobile devices or support mobile clients, the challenges identified here will become more pronounced. Client devices will provide a number of pre-installed services and users will add their own selections. Consequently, even stronger decoupling and modularization may be needed: A mobile infrastructure for mobile information services needs to flexibly support existing, changing or new services. The next design step in the TIP project will therefore see the completion of re-designing TIP into a Service-Oriented Architecture (SOA) using web services (TIP 3.0).

\section{Conclusion}

This paper discussed services in our TIP 2.9 prototype of a mobile tourist information system. TIP provides a new mobile infrastructure for cooperating information services, based on an event-based communication layer to support continually changing information. No existing systems fully address the problems of modular incorporation of and cooperation between various services in a mobile information delivery system.

In future work, we wish to extend the cooperation (and thus communication) between the provided services. We also plan to incorporate new services, such as access to external information sources, e.g., in digital libraries. This may lead to further exploration of sophisticated context-models which can be used for standardized communication between the services. We wish to support even more flexible service utilization: services may register and unregister depending on availability and capability of the mobile device. For TIP 3.0, we will employ a Service-Oriented Architecture (SOA) using web services.

\section{References}

1. G Buchanan and M Jones. Search interfaces for handheld web browsers. In Poster Proceedings of the 9th World Wide Web Conference, Amsterdam, Netherlands, September 2000.

2. Dina Goren-Bar. Overcoming mobile device limitations through adaptive information retrieval. Applied Artificial Intelligence, 18(6):513-532, 2004.

3. A. Hinze and A. Voisard. Location- and time-based information delivery in tourism. In Conference in Advances in Spatial and Temporal Databases (SSTD 2003), volume 2750 of $L N C S$, Greece, July 2003. 